\title{
RESULTS OF OBSERVATIONS OF MAXIMA OF PULSATING STARS ${ }^{1}$
}

\author{
J. H. Peña ${ }^{2,3,4}$, H. Huepa ${ }^{2}$, D. S. Piña ${ }^{3,4}$, J. Guillén ${ }^{4}$, A. Rentería ${ }^{2}$, J. D. Paredes ${ }^{5}$, R. Muñoz ${ }^{6}$, J. Donaire ${ }^{6}$, \\ and T. Benadalid ${ }^{6}$ \\ Received July 23 2020; accepted January 292021
}

\begin{abstract}
The systematic study of some HADS stars, recognized as variables for decades, has allowed us to provide data on their secular variations through $\mathrm{O}-\mathrm{C}$ analysis. However, some of the data have large gaps without observations. This is our motivation for continuously observing these stars as part of the research carried out by the "Grupo de Astronomía Observacional del Observatorio de Tonantzintla" (GAOOT). This article is our third compilation of times of maxima for pulsating stars. These observations have been carried out at the Observatorio Astronómico Nacional de Tonantzintla (TNT) and San Pedro Mártir (SPM), México and for the first time we also present data from the Complejo Astronómico de Cota Cota, Bolivia (Universidad Mayor de San Andrés) and the Observatorio Astronómico Centroamericano de Suyapa, Honduras (Universidad Nacional Autónoma de Honduras).
\end{abstract}

\section{RESUMEN}

El estudio sistemático de algunas estrellas pulsantes HADS ha permitido proporcionar datos sobre sus variaciones seculares mediante el analisis O-C. Sin embargo, en algunas de ellas los datos presentan grandes vacíos temporales sin observaciones. Esta es una motivación por la que hemos decidido observar continuamente estas estrellas como parte del trabajo que realiza el Grupo de Astronomía Observacional del Observatorio de Tonantzintla. Este grupo presenta esta tercera compilación de tiempos de máximo de estrellas pulsantes. Dichas observaciones se han llevado a cabo en los Observatorios Astronómicos Nacionales de Tonantzintla (TNT) y San Pedro Mártir (SPM), México a los que se han unido el Complejo Astronómico de Cota Cota, Bolivia (Universidad Mayor de San Andrés, Bolivia) y el Observatorio Astronómico Centroamericano de Suyapa (Universidad Nacional Autónoma de Honduras).

Key Words: ephemerides — stars: variables: Scuti

\section{MOTIVATION}

The study of $\delta$ Scuti stars has been carried out for many years since the pioneering works of Breger (1966) and Millis (1966). It was shortly after this

\footnotetext{
${ }^{1}$ Based on observations collected at the San Pedro Mártir and Tonantzintla Observatories, México, Tegucigalpa at Honduras and La Paz, Bolivia.

${ }^{2}$ Observatorio Astronómico Nacional de Tonantzintla, Universidad Nacional Autónoma de México, México.

${ }^{3}$ Instituto de Astronomía, Universidad Nacional Autónoma de México, México.

${ }^{4}$ Facultad de Ciencias, Universidad Nacional Autónoma de México, México.

${ }^{5}$ Facultad de Ciencias, Universidad Central de Venezuela, Venezuela.

${ }^{6}$ Planetario Max Schreier, Universidad Mayor de San Andrés, Bolivia.
}

that the monitoring of some of these stars was started in Mexico (Warman, Malacara and Breger, 1974, Warman, Peña and Arellano Ferro, 1979) and it has continued ever since.

More recently, with the acquisition of CCD detectors thirteen years ago, the observations of $\delta$ Scuti stars were retaken at the Observatorio Astronomico Nacional de Tonantzintla (TNT) with the participation of many students of the Observational Astronomy courses taught at the UNAM and later of the Escuela Latinoamericana de Astronomía Observacional (ESAOBELA). These students have provided us with motivation, and have supplied an eager workforce. 
TABLE 1

CHARACTERISTICS OF THE OBSERVED STARS

\begin{tabular}{|c|c|c|c|c|c|c|}
\hline ID & $\mathrm{RA}$ & Dec & Magnitude & Spectral Type & Epoch (d) & $P(d)$ \\
\hline AD Ari & 021745 & +182718.00 & 7.43 & $\mathrm{~F} 0$ & 2453330.654 & 0.53972 \\
\hline RV Ari & 021507 & +180427.91 & 11.61 & A0 & & 0.09312808 \\
\hline BE Lyn & 091817 & +460911.31 & 8.80 & A3 & & \\
\hline BL Cam & 034719 & +632242.14 & 13.03 & & & 0.03909844 \\
\hline V367 Cam & 044055 & +533806.46 & 10.47 & & & 0.121596 \\
\hline KU Cen & 115151 & $-41 \quad 1709.23$ & 13.48 & & & 0.07996 \\
\hline $\mathrm{AD}$ Cmi & 075247 & +013550.47 & 9.38 & F0IV/V & 2453478.4714 & 0.1229746 \\
\hline $\mathrm{AZ} \mathrm{Cmi}$ & 074407 & +02 2419.52 & 6.47 & A5IV & & 0.095205 \\
\hline VZ Cnc & 084052 & +094927.15 & 7.18 & A9III & & 0.178364 \\
\hline KZ Hya & 105054 & -252114.72 & 10.06 & B9III/IV & 2442516.15836 & 0.0595104212 \\
\hline AN Lyn & 091428 & +424638.36 & 10.64 & $\mathrm{~A} 7 \mathrm{IV} / \mathrm{V}$ & & \\
\hline BO Lyn & 084301 & +405951.77 & 11.49 & & & \\
\hline SZ Lyn & 080935 & +442817.61 & 9.08 & F2 & 2438124.39824 & 0.120534920 \\
\hline TV Lyn & 073331 & +474809.83 & 11.54 & A6 & 2440950.922 & 0.24065119 \\
\hline 1 Mon & 055901 & -092256.00 & 6.16 & $\mathrm{~F} 2 / 3 \mathrm{IV}$ & 2441661.1668 & 0.13612600 \\
\hline V1162 Ori & 053201 & -071524.65 & 9.9 & & & \\
\hline RR Leo & 100743 & +235930.32 & 9.94 & F0 & 2443295.402 & 0.4523933 \\
\hline V369 Sct & 185116 & -062111.16 & 9.35 & F3/5II & 2440393.709 & 0.223 \\
\hline AE Uma & 093653 & +440400.40 & 11.35 & A9 & 2435604.338 & 0.086017055 \\
\hline EX Uma & 084512 & +563626.76 & 11.02 & & & \\
\hline GW Uma & 104411 & +444044.11 & 9.89 & F3V & & \\
\hline YZ Boo & 152406 & +365200.60 & 10.36 & F0 & 2448500.0030 & 0.1040920 \\
\hline EH Lib & 145855 & -005653.01 & 9.83 & A5 & 2433438.6082 & 0.0884132445 \\
\hline
\end{tabular}

TABLE 2

TIMES OF MAXIMUM LIGHT OF THE VARIABLE STARS CONSIDERED

\begin{tabular}{|c|c|c|c|c|c|c|c|c|c|c|}
\hline ID & Date & $\mathrm{N}$ & $\Delta t(\mathrm{~d})$ & Nmax & $\begin{array}{c}\text { Tmax } \\
+2450000\end{array}$ & Telescope & Filters & Detector & Observatory & $\begin{array}{c}\text { Observers/ } \\
\text { Reducers }\end{array}$ \\
\hline AD Ari & 19120607 & 368 & 0.2119 & 1 & 8824.6543 & $\mathrm{Me}$ & $\mathrm{V}$ & 1001 & TNT & JGT,HHC/JGT \\
\hline \multirow[t]{3}{*}{ RV Ari } & 19112930 & 81 & 0.1294 & 1 & 8817.7421 & $\mathrm{Me}$ & V & 1001 & TNT & FS,DSP/DSP \\
\hline & 19113031 & 113 & 0.1841 & 2 & 8818.7621 & $\mathrm{Me}$ & V & 1001 & TNT & FS,DSP/DSP \\
\hline & 19113031 & & & & 8818.8606 & & & & & \\
\hline BE Lyn & 17032223 & 420 & 0.1173 & 1 & 7835.6745 & M8 & G & 402 & OACS & AA/ARL,GIEP \\
\hline \multirow[t]{18}{*}{ BL Cam } & 20011112 & 122 & 0.0960 & 3 & 8860.7052 & M2 & wo & andor & $\mathrm{TNT}$ & E20/HHC \\
\hline & & & & & 8860.7448 & & & & & \\
\hline & & & & & 8860.7842 & & & & & \\
\hline & 20011213 & 162 & 0.1396 & 3 & 8861.7247 & M2 & wo & andor & TNT & $\mathrm{E} 20 / \mathrm{HHC}$ \\
\hline & & & & & 8861.7641 & & & & & \\
\hline & & & & & 8861.8022 & & & & & \\
\hline & 20011314 & 116 & 0.0880 & 3 & 8862.7415 & M2 & wo & andor & TNT & E20/HHC \\
\hline & & & & & 8862.7804 & & & & & \\
\hline & & & & & 8862.8204 & & & & & \\
\hline & 20011415 & 151 & 0.1230 & 3 & 8863.7182 & M2 & wo & andor & TNT & $\mathrm{E} 20 / \mathrm{HHC}$ \\
\hline & & & & & 8863.7599 & & & & & \\
\hline & & & & & 8863.7959 & & & & & \\
\hline & 20011617 & 117 & 0.0906 & 2 & 8865.7510 & M2 & wo & andor & TNT & E20/HHC \\
\hline & & & & & 8865.7905 & & & & & \\
\hline & 20011718 & 146 & 0.1187 & 2 & 8866.7287 & M2 & wo & andor & TNT & E20/HHC \\
\hline & & & & & 8866.7684 & & & & & \\
\hline & 20022425 & 40 & 0.0819 & 2 & 8904.6927 & 84 & $u v b y-\beta$ & danish & SPM & DSP/DSP \\
\hline & & & & & 8904.7318 & & & & & \\
\hline
\end{tabular}


TABLE 2. CONTINUED

\begin{tabular}{|c|c|c|c|c|c|c|c|c|c|c|}
\hline ID & Date & $\mathrm{N}$ & $\Delta t(\mathrm{~d})$ & $N \max$ & $\begin{array}{c}\text { Tmax } \\
+2450000\end{array}$ & Telescope & Filters & Detector & Observatory & $\begin{array}{c}\text { Observers/ } \\
\text { Reducers }\end{array}$ \\
\hline V367 Cam & 20011314 & 91 & 0.1442 & 1 & 8862.8298 & M1 & $\mathrm{V}$ & ST- 8300 & TNT & E20/JGT \\
\hline \multirow[t]{4}{*}{ KU Cen } & 20011213 & 97 & 0.0818 & 1 & 8861.9195 & M2 & wo & andor & TNT & E20/HHC \\
\hline & 20011314 & 100 & 0.0724 & 1 & 8862.8795 & M2 & wo & andor & TNT & $\mathrm{E} 20 / \mathrm{HHC}$ \\
\hline & 20011415 & 120 & 0.0934 & 1 & 8863.9195 & M2 & wo & andor & TNT & E20/HHC \\
\hline & 20011617 & 137 & 0.1232 & 2 & $\begin{array}{l}8865.9165 \\
8865.9965\end{array}$ & M2 & wo & andor & TNT & E20/HHC \\
\hline \multirow[t]{14}{*}{ AD CMi } & 16021112 & 130 & 0.1400 & 1 & 7430.7541 & M2 & V & 1001 & TNT & DSP/DSP \\
\hline & 16031112 & 167 & 0.1500 & 1 & 7459.6543 & M1 & $\mathrm{V}$ & 1001 & TNT & AOA16/DSP \\
\hline & 17011415 & 144 & 0.1200 & 1 & 7768.8131 & M1 & V & 1001 & TNT & E17/DSP \\
\hline & 17120607 & 40 & 0.1000 & 1 & 8095.0522 & 84 & $u v b y-\beta$ & danish & SPM & JCC,DSP/DSP \\
\hline & 18030203 & 142 & 0.1600 & 1 & 8180.7831 & M1 & V & 1001 & TNT & AOA18/DSP \\
\hline & 18030304 & 157 & 0.1500 & 1 & 8181.7596 & M1 & $\mathrm{V}$ & 1001 & TNT & AOA18/DSP \\
\hline & 18031718 & 129 & 0.1300 & 1 & 8195.7759 & M1 & $\mathrm{V}$ & 1001 & TNT & AOA18/DSP \\
\hline & 19011415 & 84 & 0.1500 & 1 & 8498.8915 & M2 & G & ST-800 & TNT & E19/HHC \\
\hline & 19011516 & 97 & 0.1500 & 1 & 8499.8951 & M2 & G & $\mathrm{ST}-800$ & TNT & E19/HHC \\
\hline & 19011819 & 205 & 0.1600 & 1 & 8502.8419 & M2 & G & ST-800 & TNT & E19/HHC \\
\hline & 19012122 & 141 & 0.1900 & 1 & 8505.9170 & M2 & G & $\mathrm{ST}-800$ & TNT & E19/HHC \\
\hline & 19012223 & 186 & 0.2100 & 2 & $\begin{array}{l}8506.7780 \\
8506.9044\end{array}$ & M2 & $\mathrm{G}$ & ST-800 & TNT & E19/HHC \\
\hline & 19020102 & 228 & 0.2700 & 2 & $\begin{array}{l}8516.7387 \\
8516.8638\end{array}$ & M2 & wo & ST-8300 & TNT & $\mathrm{JGT} / \mathrm{JGT}$ \\
\hline & 19031617 & 187 & 0.1400 & 1 & 8559.7805 & $\mathrm{Me}$ & $\mathrm{V}$ & 1001 & TNT & AOA19/ARL \\
\hline AZ CMi & 20021920 & 51 & 0.1151 & 1 & 8899.8223 & 84 & $u v b y-\beta$ & danish & SPM & DSP/DSP \\
\hline \multirow[t]{7}{*}{ VZ Cnc } & 18031718 & 267 & 0.2107 & 1 & 8195.7696 & M1 & G & ST-800 & TNT & JGT,HHC/JGT \\
\hline & 19012122 & 211 & 0.1873 & 1 & 8505.9443 & M1 & G & $\mathrm{ST}-800$ & TNT & E19/JGT \\
\hline & 19012223 & 372 & 0.2447 & 1 & 8506.8515 & M1 & G & ST-800 & TNT & E19/JGT \\
\hline & 19121011 & 383 & 0.1198 & 1 & 8828.9646 & $\mathrm{Me}$ & $\mathrm{V}$ & ST-8300 & TNT & $\mathrm{JGT} / \mathrm{JGT}$ \\
\hline & 20022526 & 36 & 0.2352 & 1 & 8905.8573 & 84 & $u v b y-\beta$ & danish & SPM & $\mathrm{DSP} / \mathrm{DSP}$ \\
\hline & 20022627 & 49 & 0.0969 & 1 & 8906.9136 & 84 & $u v b y-\beta$ & danish & SPM & $\mathrm{DSP} / \mathrm{DSP}$ \\
\hline & 20032829 & 150 & 0.1304 & 1 & 8937.7654 & M1 & V & ST-8300 & TNT & $\mathrm{JGT} / \mathrm{JGT}$ \\
\hline \multirow[t]{9}{*}{ KZ Hya } & 17032223 & 270 & 0.7285 & 1 & 7835.7613 & M8 & $\mathrm{G}$ & 402 & OACS & AA/ARL,GIEP \\
\hline & 18021617 & 87 & 0.0919 & 2 & $\begin{array}{l}8166.7547 \\
8166.8144\end{array}$ & $\mathrm{Me}$ & $\mathrm{V}$ & 1001 & $\mathrm{TNT}$ & SBJ,ALZ/HHC \\
\hline & 19020102 & 6 & 0.0678 & 1 & 8516.9686 & M2 & G & ST- 800 & TNT & Bo19/JGT \\
\hline & 19030102 & 50 & 0.0409 & 1 & 8544.8786 & M1 & $\mathrm{V}$ & 1001 & TNT & AOA19/HHC \\
\hline & 19040506 & 1393 & 0.2109 & 3 & $\begin{array}{l}8579.6324 \\
8579.6918 \\
8579.7512\end{array}$ & M8 & $\mathrm{G}$ & 402 & OACS & Ho19/ARL,GIEP \\
\hline & 19042021 & 110 & 0.0930 & 2 & $\begin{array}{l}8594.6884 \\
8594.7480\end{array}$ & $\mathrm{Me}$ & $\mathrm{V}$ & 1001 & TNT & $\mathrm{DSP} / \mathrm{DSP}$ \\
\hline & 20012021 & 87 & 0.0892 & 1 & 8869.9210 & $\mathrm{Me}$ & $\mathrm{V}$ & 1001 & TNT & E20/DSP,HHC \\
\hline & 20031314 & 64 & 0.0565 & 1 & 8922.8845 & $\mathrm{Me}$ & $\mathrm{V}$ & 1001 & TNT & AOA20/HHC \\
\hline & 20031314 & 40 & 0.0309 & 1 & 8922.8847 & M1 & $\mathrm{V}$ & ST-8300 & TNT & AOA20,JGT/HHC,JGT \\
\hline AN Lyn & 20010910 & 394 & 0.0966 & 1 & 8859.9659 & M2 & wo & andor & TNT & $\mathrm{E} 20 / \mathrm{HHC}$ \\
\hline BO Lyn & 19011516 & 176 & 0.1419 & 2 & $\begin{array}{l}8499.7999 \\
8499.8890\end{array}$ & $\mathrm{Me}$ & $\mathrm{V}$ & 1001 & TNT & E18/JGT \\
\hline \multirow[t]{5}{*}{ SZ Lyn } & 18011213 & 90 & 0.0702 & 1 & 8131.8979 & M1 & $\mathrm{V}$ & ST-8300 & TNT & $\mathrm{E} 18 / \mathrm{HHC}$ \\
\hline & 18011516 & 165 & 0.1672 & 1 & 8134.9104 & M1 & $\mathrm{V}$ & ST-8300 & TNT & $\mathrm{E} 18 / \mathrm{HHC}$ \\
\hline & 18011617 & 176 & 0.0873 & 2 & $\begin{array}{l}8135.7517 \\
8135.8755\end{array}$ & M1 & $\mathrm{V}$ & ST-8300 & TNT & E18/HHC \\
\hline & 18012021 & 121 & 0.1053 & 1 & 8139.8537 & M1 & $\mathrm{V}$ & ST-8300 & TNT & E18/HHC \\
\hline & 19020203 & 115 & 0.1106 & 1 & 8517.8584 & M2 & $\mathrm{G}$ & $\mathrm{ST}-800$ & TNT & Bo19/JGT \\
\hline \multirow[t]{2}{*}{ TV Lyn } & 19011617 & 300 & 0.0208 & 1 & 8135.8526 & M2 & G & ST-800 & TNT & E18/HHC \\
\hline & 19020405 & 304 & 0.2884 & 1 & 8519.7864 & M2 & $\mathrm{G}$ & ST-800 & TNT & Bo19/JGT \\
\hline
\end{tabular}


TABLE 2. CONTINUED

\begin{tabular}{|c|c|c|c|c|c|c|c|c|c|c|}
\hline ID & Date & $\mathrm{N}$ & $\Delta t(\mathrm{~d})$ & Nmax & $\begin{array}{c}\text { Tmax } \\
+2450000\end{array}$ & Telescope & Filters & Detector & Observatory & $\begin{array}{c}\text { Observers/ } \\
\text { Reducers }\end{array}$ \\
\hline & 19020708 & 190 & 0.2903 & 1 & 8522.7723 & M2 & $\bar{G}$ & ST-800 & TNT & Bo19/JGT \\
\hline 1 Mon & 19020506 & 187 & 0.1481 & 1 & 8520.7220 & M2 & G & ST- 800 & TNT & Bo19/JGT \\
\hline \multirow[t]{3}{*}{ V1162 Ori } & 20011213 & 151 & 0.1118 & 1 & 8861.8051 & $\mathrm{Me}$ & V & 1001 & TNT & E20/DSP \\
\hline & 20011718 & 185 & 0.1533 & 2 & $\begin{array}{l}8866.7603 \\
8866.8368\end{array}$ & $\mathrm{Me}$ & V & 1001 & TNT & E20/DSP \\
\hline & 20022324 & 41 & 0.0940 & 1 & 8903.7422 & 84 & uvby $-\beta$ & danish & SPM & DSP/DSP \\
\hline \multirow[t]{9}{*}{ RR Leo } & 19011314 & 90 & 0.0881 & 1 & 8497.8039 & $\mathrm{Me}$ & V & 1001 & TNT & Bo19/DSP \\
\hline & 19012122 & 260 & 0.2516 & 1 & 8505.9479 & $\mathrm{Me}$ & $\mathrm{V}$ & 1001 & TNT & Bo19/DSP \\
\hline & 19012223 & 310 & 0.2804 & 1 & 8506.8542 & $\mathrm{Me}$ & V & 1001 & TNT & Bo19/DSP \\
\hline & 19013101 & 390 & 0.3257 & 1 & 8515.9003 & $\mathrm{Me}$ & $\mathrm{V}$ & 1001 & TNT & Bo19/DSP \\
\hline & 19020102 & 390 & 0.3589 & 1 & 8516.8054 & $\mathrm{Me}$ & $\mathrm{V}$ & 1001 & TNT & Bo19/DSP \\
\hline & 19020203 & 363 & 0.3211 & 1 & 8517.7120 & $\mathrm{Me}$ & $\mathrm{V}$ & 1001 & TNT & Bo19/DSP \\
\hline & 20022425 & 72 & 0.1883 & 1 & 8904.9676 & 84 & uvby $-\beta$ & danish & SMP & DSP/DSP \\
\hline & 20022526 & 36 & 0.2635 & 1 & 8905.8787 & 84 & uvby $-\beta$ & danish & SPM & $\mathrm{DSP} / \mathrm{DSP}$ \\
\hline & 20033031 & 375 & 0.3155 & 1 & 8939.8071 & M1 & V & ST- 8300 & TNT & $\mathrm{JGT} / \mathrm{JGT}$ \\
\hline V369 Sct & 19082930 & 471 & 0.1785 & 1 & 8817.7421 & $\mathrm{C} 16$ & $\mathrm{v}$ & 1001 & CotaCota & JMD/DSP \\
\hline \multirow[t]{7}{*}{ AE Uma } & 18011617 & 162 & 0.0545 & 2 & 8135.8224 & $\mathrm{Me}$ & V & 1001 & TNT & E18 \\
\hline & & & & & 8135.9041 & $\mathrm{Me}$ & $\mathrm{V}$ & 1001 & TNT & \\
\hline & 18021617 & 90 & 0.0746 & 1 & 8166.8715 & $\mathrm{Me}$ & $\mathrm{V}$ & 1001 & TNT & SBJ,ALZ/HHC \\
\hline & 18032526 & 100 & 0.0842 & 1 & 8203.8565 & M1 & G & ST- 800 & TNT & JGT/JGT \\
\hline & 19011415 & 69 & 0.1120 & 1 & 8498.8116 & $\mathrm{Me}$ & $\mathrm{V}$ & 1001 & TNT & E19/JGT,DSP \\
\hline & 19020304 & 273 & 0.1925 & 2 & 8518.7688 & $\mathrm{Me}$ & $\mathrm{V}$ & 1001 & TNT & Bo19/JGT \\
\hline & & & & & 8518.8482 & & & & & \\
\hline EX Uma & 20011617 & 135 & 0.2232 & 1 & 8865.9608 & M1 & G & ST- 8300 & TNT & E20/JGT \\
\hline \multirow[t]{5}{*}{ GW Uma } & 19020102 & 218 & 0.1738 & 1 & 8516.9623 & M1 & $\mathrm{V}$ & 1001 & TNT & Bo19/JGT \\
\hline & 19020203 & 267 & 0.2392 & 1 & 8517.7795 & M1 & $\mathrm{V}$ & 1001 & TNT & Bo19/JGT \\
\hline & 19022122 & 220 & 0.1721 & 1 & 8536.8787 & M1 & G & ST- 800 & TNT & JGT \\
\hline & 19022324 & 124 & 0.1085 & 1 & 8538.9137 & $1 \mathrm{M}$ & $\mathrm{V}$ & ST- 8300 & TNT & JGT,APC/JGT \\
\hline & 20011415 & 101 & 0.1310 & 1 & 8864.0182 & M1 & G & ST-8300 & TNT & E20/JGT \\
\hline \multirow[t]{6}{*}{ YZ Boo } & 18032829 & 120 & 0.1123 & 1 & 8206.8536 & M1 & G & ST- 800 & TNT & JGT,DSP/JGT \\
\hline & 18030304 & 90 & 0.0800 & 1 & 8181.8708 & me & $\mathrm{V}$ & 1001 & TNT & AOA18/HHC \\
\hline & 18031617 & 76 & 0.8148 & 1 & 8194.8829 & me & $\mathrm{V}$ & 1001 & TNT & AOA $18 / \mathrm{HHC}$ \\
\hline & 19042021 & 130 & 0.1172 & 1 & 8594.8027 & $\mathrm{Me}$ & $\mathrm{V}$ & 1001 & TNT & DSP/DSP \\
\hline & 20021920 & 30 & 0.0653 & 1 & 8899.9954 & 84 & uvby $-\beta$ & danish & SPM & DSP/DSP \\
\hline & 20022324 & 68 & 0.1622 & 2 & 8903.8477 & 84 & uvby $-\beta$ & danish & SPM & DSP/DSP \\
\hline \multirow[t]{2}{*}{ EH Lib } & 18031718 & 129 & 0.1114 & 2 & 8195.8240 & me & $\mathrm{V}$ & 1001 & TNT & $\mathrm{AOA} 18 / \mathrm{HH}$ \\
\hline & & & & & 8195.9122 & & & & & \\
\hline
\end{tabular}

\section{Remarks:}

1. Telescope

$1 \mathrm{M}$ - $1 \mathrm{~m}$ telescope

ME - 10" Meade LX200 telescope Equatorial

M1 - 10" Meade LX200 telescope

M2 - 10" Meade LX200 telescope

C11 - 11" Celestron telescope

$84-0.84 \mathrm{~m}$ telescope

C16 - 16" Celestron Pacific telescope

M8 - Meade LX200 telescope
2. Detector

ST8 - CCD SBIG ST-8

1001 - CCD SBIG ST-1001

8300 - CCD SBIG ST-8300

danish - uvby- $\beta$ Photometer

402 - CCD SBIG ST-402
3. Filter

V - V-filter in UBV system

G - Green in RGB set

$\mathrm{V}$ - y-filter in uvby system wo - Without filter 
Remarks (Continued). Observers.: AA: A. Artola (UNAH); ALZ: A. L. Zuñiga; ARL: A. Rentería; DSP: D. S. Piña; FS: F. Saldaña; JCC: J. Calderón; JGT: J. Guillen; JHP: J. H. Peña; SBJ: S. B. Juárez.

E17 (ESAOBELA 2017): Ramirez, Vanesa; Rodríguez, Mariana; Vargas, Stephany; Castellón, Cindy; Salgado, Ricardo; Mata, Joaquin; Santa Cruz, Raúl; Chipana, Karol; Gonzales, Lisseth; Rodríguez, Reina; De la Fuente, Diana.

E18 (ESAOBELA 2018): Calle, Carla; Huanca, Ever; Uchima, Juan Pablo; Ramírez, Raquel; Funes, Ricardo; Martinez, Juan José; Sarmiento, Karina; Cruz, Mauricio; Meza, Enith; Alvarado, Mayubell; Huaman, Victor; Ochoa, Gerson; Matamoros, Andrea.

E19 (ESAOBELA 2019): Blanco, Agustina; Benadalid, Tania; Donaire, Johany; Salazar, Luis; QuirósRojas, Marianela; Portllo, Alejandro; Escobar, Pablo; Mejía, Raquel; Mireles, Monica; León, Antonis; Zelada, Cindy; Báez, Sol-haret; Ng,Jessica.

E20 (ESAOBELA 2020): Carrasco, Laura; Vargas, Camilo; Barba, Miguel; Martinez, Glenda; Castellanos, Mitsa; Mejia, Nicole; Buenfil, Guadalupe; Vásquez, Franklin; Martínez, Bexy; Beato, Manuel; Paredes, Jhonnayker; Muñoz, Fernanda; Salazar, Azalea.

AOA16: Juarez, Karen; Lozano, Karen; Padilla, Artemio; Velázquez, Roberto; Santillan, Priscila.

AOA18: Bustos, Sergio; Carrillo, José Luis; Chávez, Brian; Navez, David; Zuñiga, Ana.

AOA19: Briones, Joshua; Castro, Celeste; Martínez, Fernanda; Posadas, Hilde; Romero, Mitzi; Soberanes, Hilkar; Velasco, Alex.

AOA20: Blas, Karla; Madrigal, Norma; Perez, Alexis; Ramirez, José; Santiago, Beatríz.

Bo19 (Bolivia 19): Benadalid, Tania; Donaire, Johany; Muñoz, Ruber.

Ho19 (OACS Honduras 2019): Artola, Adán; Meza, Maria Renee; Mejia, Luis Fernando.

GIEP: Meza, María Renee; Argueta, Christian

HADS stars provided excellent targets since they have short periods of pulsation, large amplitudes and, most of them have been observed for large time spans providing a unique chance to study their secular variations. Surprisingly, most of the observed stars we studied show evidence of a light travel-time effect, suggesting the presence of another invisible companion star.

What we have found is that some of these stars have lacked continuous observations and that there are large gaps in which they were not observed. Conscious of the need for long continuous observations, we developed a systematic monitoring of HADS stars at Tonantzintla to pursue our study of HADS double systems.

Aware of this need we have previously presented two lists of times of maxima of pulsating stars (Peña et al. 2015 and Peña et al. 2017) and here we present the third list of results from our observations.

\section{DATA COLLECTION}

This is the third compilation of OAN results of 23 variable stars obtained from 2016 to March, 2020. These observations also include some maxima from the Observatorio Astronómico Centroamericano de Suyapa, Honduras and the Complejo Astronómico de Cota Cota, Bolivia. The results are presented for 23 stars, of which 114 maxima of pulsating stars were obtained.

The CCD reduction was done with AstroImageJ (Collins et al. 2017) whereas the photoelectric observations were reduced using a classical procedure (see Peña et al., 2016 for details). All times of maxima are heliocentric and were determined with a fifth grade polynomial fitting to the light curve.

The errors were determined from the RMS error of the residuals evaluated for the times of maxima, and are about 0.016 days. The accuracy of each point is given by the exposure time and varies between $3 \mathrm{~min}$ for the 1-meter telescope and $1 \mathrm{~min}$ 
for the smaller telescopes. It may seem contradictory to use a longer integration time for the larger aperture telescope. However, this is done because the mounting of the smaller telescopes is of an altazimuth type, which does not allow long integration times. For the 1-meter telescope there were around 40,000 counts, and for the 10-inch telescope there were 11,000 counts, enough to secure the high precision desired. The photoelectric measurements and all the light curves can be requested for inspection. The procedure followed to reduce $u v b y-\beta$ of the San Pedro Martir Observatory is presented in Peña et al. (2021).

In Table 1 we present the characteristics of the observed stars; that is, their IDs, coordinates (epoch 20009), $V$ magnitude, spectral types and the observational epoch and periods in days when available from the web site of the General Catalog of Variable Stars (http://www.sai.msu.su/gcvs/ cgi-bin/search.htm GCVS) as they are reported in that source. No errors are provided. All information about telescopes, photometers and filters is specified in the remarks of the table. In Table 2 the following quantities are listed: Column 1 is the ID, Column 2, date of observation, Column 3, $\mathrm{N}$ gives the number of data points in each run, Column $4, \Delta \mathrm{t}$ is the time span in days of the run, Column 5 , the number of Tmax of the run, Column 6 , the time of maxima in HJD, Column 7, the telescope, Column 8, the filter used, Column 9, the detector, Column 10 the observatory, and finally, Column 11 gives the observers and reducers. Observers and reducers are specified in the remarks at the end of the table.

\section{REFERENCES}

Breger, M. 1966, ApJ, 146, 958

Collins, K. A., Kielkopf, J. F., Stassun, K. G., \& Hessman, F. V. 2017, AJ, 153, 77

Millis, R. L. 1966, IBVS, 137, 1

Peña, J. H., Villareal, C., Piña, D. S., et al. 2016, RMxAA, 52, 385

Peña, J. H., Renteria, A., Villareal, C. et al. 2015, IBVS, 6154,1

Peña, J. H., Renteria, A., Piña, D., \& Villareal, C. 2017, IBVS, 63, 6220

Samus, N. N., Kazarovets, E. V., Durlevich, O. V., Kireeva, N. N., \& Pastukhova, E. N. 2017, ARep, 61, 80

Warman, J., Malacara, Z., \& Breger, M. 1974, RMxAA, 1,143

Warman, J., Peña, J. H., \& Arellano Ferro, A. 1979, AJ, 84, 109

T. Benadalid, J. Donaire, and R. Muñoz: Planetario Max Schreier, Universidad Mayor de San Andrés, Bolivia.

J. Guillén, J. H. Peña, and D. S. Piña: Facultad de Ciencias, Universidad Nacional Autónoma de México, México.

H. Huepa, J. H. Peña, and A. Rentería: Observatorio Astronómico Nacional de Tonantzintla, Universidad Nacional Autónoma de México, México.

J. D. Paredes: Facultad de Ciencias, Universidad Central de Venezuela, Venezuela.

J. H. Peña and D. S. Piña: Universidad Nacional Autónoma de México, Instituto de Astronomía, AP 70-264, CDMX 04510, México (jhpena@astroscu.unam.mx). 